\title{
Transfusion Associated Hyperkalemia and Cardiac Arrest in an Infant after Extracorporeal Membrane Oxygenation
}

\author{
Do Wan Kim, M.D., Kyeong Ryeol Cheon, M.D. , Duck Cho, M.D. ${ }^{\dagger}$, Kyo Seon Lee, M.D., Hwa Jin Cho, M.D., , \\ and In Seok Jeong, M.D.
}

Department of Thoracic and Cardiovascular Surgery, ${ }^{*}$ Department of Pediatrics, Chonnam National University Hospital, Gwangju;

'Deparment of Laboratory Medicine and Genetics, Samsung Medical Center, Seoul, Korea

\begin{abstract}
Cardiac arrest associated with hyperkalemia during red blood cell transfusion is a rare but fatal complication. Herein, we report a case of transfusion-associated cardiac arrest following the initiation of extracorporeal membrane oxygenation support in a 9-month old infant. Her serum potassium level was increased to $9.0 \mathrm{mEq} / \mathrm{L}$, soon after the newly primed circuit with pre-stored red blood cell (RBC) was started and followed by sudden cardiac arrest. Eventually, circulation was restored and the potassium level decreased to $5.1 \mathrm{mEq} / \mathrm{L}$ after $5 \mathrm{~min}$. Extracorporeal membrane oxygenation (ECMO) priming is a relatively massive transfusion into a pediatric patient. Thus, to prevent cardiac arrest during blood-primed ECMO in neonates and infants, freshly irradiated and washed RBCs should be used when priming the ECMO circuit, to minimize the potassium concentration. Also, physicians should be aware of all possible complications associated with transfusions during ECMO.
\end{abstract}

Key Words: blood transfusion; extracorporeal membrane oxygenation; heart arrest; hyperkalemia.

Extracorporeal membrane oxygenation (ECMO) is a well-established treatment for patients with severe cardiopulmonary failure. On ECMO commencement, a large amount of priming liquid from the extracorporeal circuit enters the body, equivalent to a rapid and massive transfusion. Caution is required as this increases the risk of transfusion-associated hyperkalemia and associated cardiac arrest.[1] Moreover, when an infant is undergoing ECMO, the use of irradiated pre-stored packed red blood cell (RBC) to prime ECMO support may increase the risk of transfusion-associated hyperkalemic cardiac arrest (TAHCA). Herein, we report the case of an infant, who experienced sudden cardiac arrest after EMCO initiation.

\section{Case Report}

A 9-month-old infant was admitted for treatment of severe pulmonary hypertension. She was born at gestational age 27

Received on March 12, 2015 Revised on May 6, 2015

Accepted on May 13, 2015

Correspondence to: Hwa Jin Cho, Department of Pediatrics, Chonnam National University Hospital, 42 Jebong-ro, Dong-gu, Gwangju 501-757, Korea

Tel: +82-62-220-6646, Fax: +82-62-222-6103

E-mail: chhj98@gmail.com

*No potential conflict of interest relevant to this article was reported. weeks, and her birth weight was $700 \mathrm{~g}$. Her history included respiratory distress syndrome and bronchopulmonary dysplasia. She was discharged from the neonatal intensive care unit at 3 months of age, with no evidence of pulmonary hypertension upon transthoracic echocardiography (TTE) and no relevant symptoms. At 9 months of age, her mother noted new cough, sputum production, and sweating. TTE revealed 
severe pulmonary hypertension, tricuspid regurgitation (velocity of $4.8 \mathrm{~m} / \mathrm{s}$ ), and overloaded right ventricular (RV) pressure. She was admitted and taken on sildenafil medication. However, after 3 days, the dyspnea and hypoxia intensified, and she was transferred to the intensive care unit. She underwent intubation and ventilation, and inhaled nitric oxide (NO) was initiated at $20 \mathrm{ppm}$. However, her condition became increasingly aggravated. Consequently, venovenous (VV)-ECMO was initiated via a central cannulation to treat the severe hypoxia. Catheters were inserted directly into the right atrium and pulmonary artery through $16 \mathrm{Fr}$. and 12 Fr. venous drainage cannulas (Edwards Lifesciences Research LLC, Irvine, CA, USA). Two days after ECMO support commenced, we ordered the transfusion of two packs of packed RBCs that had been $\gamma$-irradiated 19 hours prior, and no specific complication developed during transfusion. On day 4 of ECMO support, the oxygenator failed and required the change of ECMO circuit. The circuits were newly primed with pre-stored RBC that has been $\gamma$-irradiated 3 days prior to priming. However, immediately after ECMO was restarted, her heart rate dropped from 120 to $30 \mathrm{bpm}$. Soon thereafter, the electrocardiogram showed flattening, and cardiopulmonary resuscitation (CPR) was commenced. At the beginning of $\mathrm{CPR}$, serum potassium level was increased to $9.0 \mathrm{mEq} / \mathrm{L}$ by arterial blood gas analysis (Gem Premier 3500, Werfen Company, Instrumentation Laboratory, Bedford, MA, USA). And then calcium gluconate was immediately infused intravenously, then RI 50U and continuous renal replacement therapy was started. Eventually, circulation was restored, and the potassium level decreased to $5.1 \mathrm{mEq} / \mathrm{L}$ after $5 \mathrm{~min}$ by arterial blood gas analysis and to $4.2 \mathrm{mEq} / \mathrm{L}$ after $30 \mathrm{~min}$ in blood laboratory test. We confirmed that potassium level in stored unit was $35.0 \mathrm{mEq} / \mathrm{L}$ in blood laboratory test. However she survived the hyperkalemic cardiac arrest, she died of her underlying condition after 13 days.

\section{Discussion}

In pediatric patients, the extracorporeal volume of the ECMO circuit comprises a large proportion of the total blood volume, and blood products are ideally used upon ECMO priming. However, when ECMO begins, a large volume of liquid from the extracorporeal circuit enters the pa- tient's circulation, equivalent to a rapid and massive transfusion, associated with all possible relevant complications.

Lymphocytes in transfused blood can proliferate and engage in immunological attack, triggering graft-versus-host disease (GVHD).[2] Currently, the only method used to prevent transfusion-associated GVHD is irradiation. RBCs transfused into neonates or patients with poor immunity are frequently irradiated to prevent possible GVHD.[3]

Usually, irradiated cells function normally. However, their shelf-life is short and potassium leakage rapid.[4] Furthermore, packed RBCs are usually stored in the cold to prevent bacterial growth. Low temperature may damage the membranes of RBCs, compromising pump function, in turn triggering potassium leakage into the extracellular space. Ultimately, such leakage is accelerated by irradiation.[4] Although filtered irradiated RBCs can be used up to 28 days after irradiation, hyperkalemia can be a life-threatening concern even $24 \mathrm{~h}$ after irradiation, especially in patients who require large volumes of transfusion fluids, such as those on ECMO.

Transfusion-induced cardiac arrest is more common in young infants than in older patients. The risk factors include rapid massive transfusion and renal dysfunction.[1] Various methods are used to minimize the problem. As extracellular potassium concentrations in suspensions of washed RBCs increase with the time since irradiation, this time should be minimized unless the RBCs are washed after irradiation and this may reduce the potassium concentration.[5] Rapid transfusion should be avoided, especially through a central venous line. Transfusion through a central line is also a risk factor, because cold hyperkalemic blood can slow the SA and $\mathrm{AV}$ nodes, rendering cardiac output low.[6]

ECMO priming is a relatively massive transfusion into a pediatric patient. Thus, to prevent cardiac arrest during blood-primed ECMO in neonates and infants, freshly irradiated and washed RBCs should be used when priming the ECMO circuit, to minimize the potassium concentration. Also, physicians should be aware of all possible complications associated with transfusions during ECMO; careful monitoring is essential.

\section{References}

1) Smith HM, Farrow SJ, Ackerman JD, Stubbs JR, 
Sprung J: Cardiac arrests associated with hyperkalemia during red blood cell transfusion: a case series. Anesth Analg 2008; 106: 1062-9

2) Brugnara $\mathrm{C}$, Churchill WH: Effect of irradiation on red cell cation content and transport. Transfusion 1992; 32: 246-52.

3) Sloan SR: Neonatal transfusion review. Paediatr Anaesth 2011; 21: 25-30.

4) Weiskopf RB, Schnapp S, Rouine-Rapp K, Bostrom A, Toy P: Extracellular potassium concentrations in red blood cell suspensions after irradiation and washing. Transfusion 2005; 45: 1295-301.

5) Sohn HM, Park YH, Byon HJ, Kim JT, Kim HS, Kim CS: Application of the continuous autotransfusion system (CATS) to prevent transfusion-related hyperkalemia following hyperkalemic cardiac arrest in an infant -A case report. Korean J Anesthesiol 2012; 62: 281-4.

6) Luban NL, Leikin SL: Jehovah's Witnesses and transfusion: the pediatric perspective. Transfus Med Rev 1991; 5:253-8. 